\title{
Doctors' and nurses' views on patient care for type 2 diabetes: an interview study in primary health care in Oman
}

\author{
Nadia M. Noor Abdulhadi ${ }^{1,2}$, Mohammed Ali Al-Shafaee ${ }^{3}$, Rolf Wahlström ${ }^{4,5}$ and Katarina Hjelm ${ }^{6}$ \\ ${ }^{1}$ Department of Public Health Sciences, Division of Global Health (IHCAR), Karolinska Institutet, Stockholm, Sweden \\ ${ }^{2}$ Department of Health Affairs, Ministry of Health, Muscat, Oman \\ ${ }^{3}$ Associate Professor, Department of Family Medicine and Public Health, College of Medicine and Health Sciences, \\ Sultan Qaboos University, Muscat, Oman \\ ${ }^{4}$ Associate Professor, Department of Public Health Sciences, Division of Global Health (IHCAR), Karolinska Institutet, \\ Stockholm, Sweden \\ ${ }^{5}$ Department of Public Health and Caring Sciences, Family Medicine and Clinical Epidemiology, Uppsala University, \\ Uppsala, Sweden \\ ${ }^{6}$ Professor, School of Health and Caring Sciences, Linnaeus University, Växjö, Sweden
}

\begin{abstract}
Aim: This study aimed at exploring the experiences of primary health-care providers of their encounters with patients with type 2 diabetes, and their preferences and suggestions for future improvement of diabetes care. Background: Barriers to good diabetes care could be related to problems from health-care providers' side, patients' side or the healthcare system of the country. Treatment of patients with type 2 diabetes has become a huge challenge in Oman, where the prevalence has increased to high levels. Method: Semistructured interviews were conducted with 26 health-care professionals, 19 doctors and seven nurses, who worked in primary health care in Oman. Qualitative content analysis was applied. Findings: Organizational barriers and barriers related to patients and healthcare providers were identified. These included workload and lack of teamwork approach. Poor patients' management adherence and influence of culture on their attitudes towards illness were identified. From the providers' side, language barriers, providers' frustration and aggressive attitudes towards the patients were reflected. Decreasing the workload, availability of competent teams with diabetes specialist nurses and continuity of care were suggested. Furthermore, changing professional behaviours towards a more patient-centred approach and need for health education to the patients, especially on self-management, were addressed. Appropriate training for health-care providers in communication skills with emphasis on self-care education and individualization of care according to each patient's needs are important for improvement of diabetes care in Oman.
\end{abstract}

Key words: culture; Oman; patient-provider interaction; professional behaviour; qualitative content analysis

Received 18 February 2012; accepted 23 October 2012; first published online 21 December 2012

\section{Background}

Diabetes is developing into a global public health problem, particularly increasing in countries within

Correspondence to: Dr Nadia M. Noor Abdulhadi, Department of Public Health Sciences, Division of Global Health (IHCAR), Karolinska Institutet, SE-171 77, Stockholm, Sweden. Email: nadia.abdulhadi@ki.se the Gulf region in the Middle East (Whiting et al., 2011). Type 2 diabetes is to a great extent preventable, and its burden can be decreased worldwide through training of health-care workers to focus on people-centred care through information and empowerment of the population in general, to become aware of the importance of health promotion activities and improvement of quality

(C) Cambridge University Press 2012. The online version of this article is published within an Open Access environment subject to the conditions of the Creative Commons Attribution-NonCommercial-ShareAlike licence $<$ http://creativecommons.org/licenses/ by-nc-sa/3.0/>. The written permission of Cambridge University Press must be obtained for commercial re-use. 
and responsiveness of the health-care sector (World Health Organization, 2007).

In the Sultanate of Oman, the changes brought about by demographic and epidemiologic transition since the 1970s, have had a profound impact on the health pattern. In addition, the changing lifestyle of the Omani population towards less physical activity and less healthy dietary habits with consumption of high amount of fast food, high in refined sugar and saturated fat, have contributed to a marked increase in the prevalence of type 2 diabetes up to $12.3 \%$ in 1.9 million Omani citizens (Ministry of Health (MoH), 2008; World Health Organization, 2010).

The $\mathrm{MoH}$ is the main health-care provider in Oman and has built a modern national system offering universally accessible health services free of charge in all regions through a network of health facilities, including primary care, secondary and tertiary care levels (World Health Organization, 2010). Primary health-care centres (PHCCs) are the entry point for most patients, including patients with diabetes. Patients can be referred to secondary health care and tertiary care hospitals for specialized care (World Health Organization, 2010).

Health systems with strong primary health care are more likely to give greater attention to the management of people with diabetes in teams, including specialized diabetes care nurses who share some of the physicians' responsibilities (Peters et al., 2001; Renders et al., 2001). However, in spite of the economic and health services development in Oman, this model has not yet been established.

The clinical services for diabetes care in Oman are provided mainly in the PHCCs by general practitioners (GPs), as well as practice nurses, pharmacists and laboratory technicians. In many PHCCs, there are also dieticians and health educators, but in insufficient numbers at the time of data collection. Their role is to provide health and nutritional education for patients with diabetes if referred to them by the doctors. Complicated cases of diabetes are referred to secondary or tertiary care facilities $(\mathrm{MoH}, 2003)$. Patients with diabetes are seen with appointments in diabetes clinics in the PHCCs, and the number of appointed patients might go beyond 30 patients per clinic day for one doctor.

The quality of care for patients with type 2 diabetes remains suboptimal worldwide regardless of the country's level of development, efficacious treatments available, health-care system or population characteristics (Engelgau et al., 2003). Furthermore, good patient-provider interactions and communication skills have been linked in empirical studies to favourable patient outcomes, such as satisfaction and recall, adherence, symptom resolution and better quality of life (Beck et al., 2002).

Patient-provider interaction is also affected by the social and cultural background of the patient (Helman, 2007). Previously, patients' beliefs about health and illness have been shown as essential determinants for self-care practice and care-seeking behaviour among patients with diabetes (Hjelm et al., 1999; 2003; 2005).

In this context, the influence of culture on the Omanis' behaviour and beliefs with regard to health issues and nutrition should be considered. Traditionally, sharing of meals with one's family and neighbours is a highly valued social interaction (Insight Guides, 2009). Consumption of dates served with coffee remains a symbol of Omani hospitality, and dates are taken frequently during the day, as in other Gulf States. There is a strong cultural and religious belief about its nutritional and economic value and it is considered as a blessing fruit according to the holy Qur'an (Lawrence, 2001).

It was concluded in previous explorative studies in PHCCs in Muscat (Abdulhadi et al., 2006; 2007) that the interactions between health-care providers and their patients with type 2 diabetes were suboptimal and there was poor collaboration between different kinds of health-care staff working in diabetes care. Furthermore, the patients expressed their dissatisfaction with issues related to the organization of the diabetes clinics, and they perceived that the doctors and nurses were neither experts nor competent in diabetes care. However, no published studies have focused the perceptions of the health-care professionals on diabetes care in Oman. Hence, this study aimed at exploring the experiences of primary health-care providers of their encounters with patients with type 2 diabetes, and their preferences and suggestions for future improvement of diabetes care.

\section{Methods}

\section{Design}

Given the exploratory nature of the study, a qualitative approach (Patton, 2002) was chosen, and semi-structured interviews were conducted. 
Table 1 Demographic characteristics of the participants

\begin{tabular}{lcc}
\hline Variable & Doctors $(n=19)$ & Nurses $(n=7)$ \\
\hline Sex & & \\
$\quad$ Females & 8 & 7 \\
$\quad$ Males & 11 & 0 \\
Age: median (range) & $40(29-55)$ years & $30(25-40)$ years \\
Arabic speaking & 11 & 5 \\
Non-Arabic speaking & 8 & 2 \\
\hline
\end{tabular}

\section{Subjects and settings}

A total of 26 health-care professionals (19 doctors and 7 nurses), aged 25-55 years, working in diabetes care at PHCCs were interviewed in 2006 (see Table 1). We purposely aimed at interviewing the same health-care professionals (23 doctors and 13 nurses), who had participated in a preceding observational study (Abdulhadi et al., 2006), but 10 providers (four doctors and six nurses) could not be enrolled, as they had either been transferred to other areas or were on annual leaves. Both the doctors and the nurses had more than three years of work experience in health care.

The doctors had attended at least 10 sessions of training in diabetes care with a diabetologist, which was in accordance with regulations of the $\mathrm{MoH}(\mathrm{MoH}, 2003)$. There were no specialist diabetes nurses; instead, the nurses who are responsible for provision of care for patients with diabetes and who are frequently available in diabetes clinics in the health centres are called diabetes practice nurses or diabetes educators. They have received a short (one week) training in diabetes care and education $(\mathrm{MoH}, 2003)$.

\section{Data collection}

An interview guide (Appendix 1) was developed by the first (N.A.) and third author (R.W.) on the basis of results from previous studies (Abdulhadi et al., 2006; 2007). The guide was discussed and agreed by the research team, peerreviewed by six heads of PHCCs and thereafter modified. The following key areas were explored: description of the encounters and discussions with type 2 diabetes patients; experienced difficulties in interactions and communications in general; and suggestions for future improvement of the interaction and care. The interviews were conducted by a physician (first author) and took place in the health-care providers' offices in the health centres with an average duration of 1 hour for each interview. Non-Arabic speaking doctors and nurses were interviewed in English $(n=10)$, and other participants could choose between their mother tongue Arabic ( $n=5$ nurses) or English $(n=11$ doctors). All interviews were audiotaperecorded with the participants' consent and transcribed verbatim.

\section{Data analysis}

Qualitative content analysis was applied inductively (Patton, 2002). The transcripts were read through several times to obtain a good sense of the whole text. The text was then divided into meaning units, which were labelled with codes and abstracted. The various codes were compared on the basis of differences and similarities and sorted into categories and subcategories (see Appendix 2).

The first author conducted the analysis and the content in the categories were primarily checked by the last author and discussed with all authors, each of them with qualifications in public health sciences and qualitative research. Disagreements were resolved through discussions between the authors until consensus was reached. Findings are presented as categories and subcategories with illuminative quotations to enhance credibility (Patton, 2002).

\section{Ethical approval}

Ethical approval was obtained from the Medical Research and Ethics Committee of Oman, and the study was conducted in accordance with the Helsinki Declaration (World Medical Association, 2009). Verbal consents from the participants in the interviews were obtained after explanation of the study objectives and implementation, and their anonymity was guaranteed.

\section{Findings}

Three main factors were considered as barriers affecting diabetes care, related to the organization of the diabetes clinics, the patients and the health-care providers. These factors, with subcategories, will be presented first, followed by suggestions to improve diabetes care. 


\section{Organizational factors}

\section{Workload}

High workload was mentioned by the participants as a major problem affecting the interactions with the patients. They added that only one doctor runs the diabetes clinic and has to consult a big number of patients that might go beyond 30 patients during the seven official working hours in most of the health centres.

It is a burden on one doctor to see 30 or more patients, we had to do a lot of things to each patient in addition to documentation of the findings in the computer.

$$
\text { (Doctor (D)13) }
$$

The doctors added that sometimes they had to finish too quickly in order to avoid reactions of irritation and interruptions from other patients, who had been waiting for long time to see the doctor.

The major factor is the stress I get when the other patients started shouting outside, knocking the door and asking when they will see the doctor which forced me to finish quickly with the consultation.

The reason for giving appointments to several patients at the same time was justified by the total large number of registered patients in each catchment area, and therefore the policy is to see each patient at least every two or three months or more frequently, as several patients had poorly controlled diabetes.

\section{Lack of teamwork approach}

The doctors said they had to do everything for the patients due to lack of diabetes specialist nurses, and added that the available diabetes practice nurses were neither well trained nor qualified in provision of diabetes care. Furthermore, there was a shortage in the number of dieticians and health educators, and no podiatrists for foot care.

Dietician and health educator are not here every time, our nurses are not well trained, not qualified and do not know the process of care for diabetic patients. Well some few nurses are good but in general we do not have specialist diabetes nurses in Oman, neither chiropodists, doctors do all the job.

The nurses added:

We do not have time to provide health education, the doctors do.

(Nurse (N)2)

Some doctors expressed that there was lack of trust from their side in the competency of the available health-care professionals at the clinics.

"Our nurses do not give positive advices to the patients, the health educators and dieticians lack good knowledge, I do not trust them all. I do everything myself.

However, other doctors expressed their satisfaction with diabetes care and health outcomes, as they were working in a team, and they did not criticize the competences of the nurses or the other medical staffs.

In our health centre we interact with the patients as a team including dietician, nurse and doctor. Most of our diabetic patients are $O K$ and well controlled.

\section{Patient factors}

\section{Poor patient adherence}

The participants were, in general, dissatisfied with the patients for their poor adherence to healthy diet, exercise and medicines, including refusal of insulin and reluctance to be referred to secondary or tertiary care. They specifically blamed the elderly and less educated, who were perceived as difficult patients, as they often showed no interest and no willingness to listen to the doctor or attend health education.

The poor adherence to healthy diet was related to eating of unhealthy traditional food, in addition to the habit of consumption of large quantities of dates, which were considered as a source of energy, health and well-being. The doctors and nurses found it difficult to convince the patients to omit or decrease the consumption of dates. In addition, the Omanis consume the local sweets (halwa), which mainly have a high content of fat, nuts and sugar.

To modify patients' diet is a real problem, one patient was angry and said he will eat 
what he wants and asked me what my grandfathers used to eat long time, they used to depend on dates mainly and the Omani halwa and their health was perfect.

Furthermore, the doctors and nurses expressed that older patients or patients with poor reading skills have difficulties with diet control, whereas the young and educated patients appear to make efforts to change their traditional eating behaviour.

You know he is an old man and it is not easy for an old man to change his mind and habits absolutely and same for the uneducated ones.

The doctors added that most of the patients with diabetes, regardless of their age or gender, did not practice physical activities mostly because of leg pain or arthritic problems or low motivation among old or even not so old patients, because they can rely on transport support by their families.

Her diet was quite appropriate, but her exercise was really not there. She hardly does anything and depends on her family. Also a lot of patients do not exercise due to joint or leg pains.

Poor medication adherence, in general, including increasing doses and refusal to use insulin was expressed to be mainly because of the patients' fear of harm or damage to body organs. The doctors particularly expressed that they experienced difficulties to convince the patients to use insulin to save their lives, and that diabetes complications are related to poor glycaemic control and not to the insulin.

To use the medicines appropriately is a problem, to add or increase the medication dose is another problem, and to start insulin is a real problem. It seems that there are some beliefs among patients that if they are on insulin that is the end, it's like a terminal case and insulin might harm them.

However, a few doctors mentioned that they also had responsibility to ensure that the patients received the most appropriate treatment, including insulin when indicated.

May be there must be a change in our way of explaining, we are making the patients afraid of insulin, I feel there is something wrong with our way of counseling.

The doctors said that many patients preferred to use certain herbs to treat diabetes and they related their blood sugar control to these herbs only and not to the prescribed hypoglycaemic agents.

Poor cooperation of some patients, especially the elderly, concerning referral to secondary or tertiary care for annual eye check-up or for screening of complications, was also mentioned as a difficulty to good diabetes care.

I have four patients who completely refused to go for eye check-up because they find it too far away from their living places. They prefer to be referred to the nearest hospital due to transport costs.

Yes I face difficulties, the elderly patients refuse to go for eye check-up as some think that any surgical intervention with their eyes will lead to blindness.

\section{Patients' beliefs about illness}

The participants expressed that beliefs about diabetes among Omani patients are constructed out of cultural values and spiritual beliefs. Several patients were said to believe that any illness including diabetes comes from Allah (God). Allah decides their fate and their time for death with or without having diabetes, and that they live only one life and die once by the willpower of Allah only, and there will be no prolongation of their lives whether they care about their health or not. These beliefs made them less likely to follow the medical advices, less persuaded for self-care behaviour or changing their unhealthy lifestyle.

No use, he said everything comes from Allah, and no one will live longer than what is supposed to be so why to make efforts or to change their style of living. 
On the other hand, both doctors and nurses mentioned that many patients feared diabetes, as it was perceived a disaster and a potential threat to life. They feared complications like blindness, kidney damage, leg amputations and death. Furthermore, some doctors mentioned they had experienced difficulties with some patients, regardless of their education or age, who refused even the registration in the national diabetes register and insisted to share the same dishes during both family meals and social occasions in order not to feel stigmatized.

A young educated patient was shy to tell his family and friends about his condition and refused to be registered in the diabetes register and refused to eat separate meals. I discussed with him that diabetes is not a stigma and that he should tell his family to help him control his diet.

\section{Factors related to health-care providers}

The doctors acknowledged themselves for their efforts towards their patients with diabetes to improve their health through frequent health education, including education about complications, exercise and mental stress and provision of optimal care, including prescription of suitable medicines and sometimes the maximum medicine dose if needed. However, some barriers to good care on the doctors' and nurses' side were identified.

\section{Doctors' and nurses' frustration with non-adherent patients}

Frustration due to unsuccessful efforts to make the patients adherent to medical advices and instructions was mentioned by doctors and nurses. They used expressions like 'the patients are not listening', 'not understanding' or 'not following the instructions'.

Some doctors perceived the discussions with certain patients as useless and they preferred to behave with them as disease-oriented doctors.

No, I did not ask about any personal details, what is the use of that. She never follows any instructions. I focused only on her medical condition and that is it.

\section{Doctors' attitudes}

Some doctors mentioned that they expressed aggression towards the non-adherent patients and sometimes they frightened them with the potential complications of diabetes. They even said that they stopped prescribing medicines because these patients did not use them.

Sometimes I scare the non-cooperative patients otherwise they will not listen. I was aggressive with one ignorant lady and told her in the last visit that to give you the medicine is just a waste of resources. I didn't prescribe any medicine. She was surprised.

In contrast, other doctors emphasized on good communication and relations with their patients, even with the risk of being medically inaccurate, and assured that this created trust and confidence in addition to improving patients' adherence. Those doctors denied any difficulties in interactions or in management of diabetes.

Usually no, not at all, I didn't face any difficulties. My patients trust me. This is because my approach is friendly which is very important for good interaction.

\section{Communication problems related to language}

The non-Arabic speaking doctors expressed language problems with patients who did not speak English. These doctors usually turned to the family members or asked for assistance of other health professionals from the local community to translate the medical information. They avoided deep discussions or social talk with the patients and focused only on the current medical condition.

No, because of my broken Arabic I avoid any deep discussion or social talk, I focus only on the disease.

\section{Suggestions to improve diabetes care}

Organizational efficiency of diabetes clinics

The doctors and nurses addressed the need for organizational improvement of the diabetes clinics through less number of patients to enable better 
interactive communication during the medical interview. The doctors emphasized the importance of continuity of care with their patients.

It is very important to see less number of patients I think ten to fifteen is reasonable. It is also important to maintain continuity of care as much as possible. If there is something in my mind for this patient, what I am going to change today or tomorrow and so.

The doctors and nurses addressed the need for teamwork and to strengthen the role of the nurses in diabetes care.

I suggest giving our nurses some responsibilities as everything is on the head of the doctors. We want the nurses to be able to evaluate the conditions of the patients then to decide who needs to see the doctor and when.

\section{Improvement of health-care professionals' behaviours}

Some doctors mentioned that there should be 'a personal interest' of health-care providers in diabetes care and to show this interest to the patients. Furthermore, they suggested avoiding giving instructions to the patients, but instead have good communication and respect their concerns, as this would be a more useful way to correct the patients' understanding of diabetes and gain their cooperation.

\section{Self-management and health education}

The doctors and nurses addressed the need for education to the patients on self-management and self-monitoring behaviour.

Promotion of self-monitoring behaviour is important and cost effective. A lot of health education is needed as our patients do not know about the disease and how to care for themselves.

Health education to the patients in groups, rather than individually, using audio-visual aids and attractive health education leaflets was also suggested and considered as important for improvement of patients' knowledge and understanding about diabetes. Furthermore, it was suggested that health education should include the families and the whole community through media, newspapers, television, and in the schools and mosques.

\section{Discussion}

Our findings expose several barriers to good diabetes care in the urban region of Oman, in spite of the health-care system development. Weaknesses in organization of diabetes clinics and barriers related to patients and health-care professionals were brought to the fore.

\section{Organization of diabetes clinics and suggestions for improvement}

High workload associated with short consultations was reflected in addition to lack of teamwork and criticism of the competency of diabetes practice nurses, dieticians and health educators. However, defining the optimal size of a practice is complex, as views of doctors, patients and health service managers may differ (Campbell et al., 2001). Nevertheless, good doctor-patient communication inevitably takes more time, and compression of consultation length possibly affects good diabetes control and increases the risk of developing costly complications related to diabetes (Sackett, 1997).

Some doctors related the lack of teamwork approach to mistrust of the competences of the available nurses, dieticians and health educators. The complexity of diabetes care requires a diffusion of responsibility for the care from physicians to nurses, dieticians and patients, at best carried out within a team (Donnelly and Anderson, 1990). Furthermore, there is now considerable evidence that involvement of specialized nurses trained in diabetes care yields better results than traditional physician-led care, as in countries like Sweden, England and the Netherlands where primary care is based largely on multi-professional teams (World Health Organization, 2008). Our findings support development of such teams and models of care at PHCCs in Oman, and should be considered by health policy makers.

The doctors also emphasized the importance of continuity of care for their overall performance and relations with their patients. Provider-patient continuity in primary care has been associated 
with better glycaemic control among patients with type 2 diabetes (Parchman et al., 2002).

\section{Patients' barriers and culture}

The poor adherence to treatment, mainly among elderly and less educated patients, was perceived as related to patients' beliefs on the basis of habits, traditions and lack of knowledge about diabetes and its management. Furthermore, the health-care staff expressed that many patients showed insufficient self-efficacy and instead reliance on God (Allah) and his destiny, with regard to their diabetes. The influence of religion as a societal order is important to consider in health care irrespective of whether a person is a believer or not (Hjelm et al., 2003). Although most Muslims appreciate modern medicine and will seek appropriate health care, the belief in predestination may lead some patients to not comply with treatment plans or seeking care (Lawrence and Rozmus, 2001). Health-care providers need to consider such cultural and religious beliefs in their encounters with patients with diabetes (Hjelm et al., 2003; 2005; Helman, 2007). Reasons for not adhering to prescribed diabetes medicines could be related to poly-pharmacy and multiple daily dosing (Cramer, 2004), which may create confusion regarding the drug regimen, in addition to fears of side effects and the progressive nature of the disease (Puder and Keller, 2003).

Other perceived barriers were in general poor health literacy and cultural beliefs about insulin or fear of insulin, which could also negatively impact the patient-provider relationship (Peyrot et al., 2005; Campos, 2007). It was also mentioned that some patients seemed to prefer herbal treatment instead of modern medicines, which could be related to fear of side effects. It has been found in other studies that general practitioners and nurses seem to delay insulin therapy until absolutely necessary, whereas specialists and other medical opinion leaders are less likely to do so (Peyrot et al., 2005). It has also been found that herbal treatment of diabetes is preferred in many countries due to lesser side effects and low cost (Modak et al., 2007).

The doctors and nurses suggested increased teamwork, improved patient education and a patient-centred approach, including good communication skills. This is in line with suggestions from other scholars that, instead of changing the medication or blaming the patients, diabetes team members should consider support to the patients through education on self-care, using good communication skills and understanding cultural beliefs (Cramer, 2004; Peyrot et al., 2005; Wens et al., 2005; Campos, 2007).

Lack of knowledge about eye complications related to diabetes and fear, particularly of laser treatment, was perceived as a great barrier, deterring some patients from attending eye clinics (Lewis et al., 2007). There are many factors predisposing to non-attendance to referral ranging from age of the patient, health beliefs, understanding of the disease, attitudes of health professionals, organization of the clinic and the financial costs of attendance (Griffin, 1998; Dalewitz et al., 2000). Providing more education to patients about diabetes retinopathy, using good communication skills and making eye clinic attendance more convenient for patients, may increase the number of patients with diabetes who accept regular eye examinations (Lewis et al., 2007).

Health education to the patients, preferably in groups rather than individual sessions, was suggested by both the doctors and the nurses. It has been reviewed that group-based training especially for self-management strategies among people with type 2 diabetes is effective in improving metabolic control (Deakin et al., 2005). Our findings emphasize the importance of recognizing and assessing individual beliefs about health, illness and medications in educational efforts to empower patients' participation in self-care (Hjelm et al., 2003).

\section{Barriers related to health-care providers}

The main factors related to doctors and nurses were their expressed frustration due to patients' poor adherence and suboptimal health outcomes; the doctors' negative attitudes towards nonadherent patients; and language problems of nonArabic speaking doctors. Studies have shown that language concordance between patients and physicians, shared understanding of advice (Rhodes and Nocon, 2003) and availability of interpreters are elements of support in the consultation and are associated with decreased communication errors, increased patient satisfaction and adherence with medications and follow-ups (Rhodes and Nocon, 2003; Karliner et al., 2007). 
The negative attitudes of some doctors towards their patients could be related to their frustration and dissatisfaction with the outcomes. It has also been shown in other studies that the patient's own expectations with regard to diabetes and health do not always correspond with the objectives and expectations of the physician's treatment proposals, and even further that physicians' ambitions to achieve good results may be in conflict with the patients' motivation to lead their own lives (Wens et al., 2005). In this respect, it is important to practice highly individualized care in diabetes management, considering cultural values, beliefs, knowledge and expectations (Hjelm et al., 2003).

To reduce doctors' and nurses' frustration, it has been suggested to shift to a model of patient-provider partnership with mutual agreement on shared decisions and closer relationship between the patient and the doctor (Wens et al., 2005). Moreover, improvement of organizational efficiency of diabetes clinics in Muscat, such as reducing the workload and number of visits of patients, will most probably positively affect the health-care providers' performance and attitudes (Campbell et al., 2001; Rethans et al., 2002).

\section{Methodological considerations}

This study had an explorative aim, and the main goal in qualitative research is to understand reality and gain information about issues or situations of central importance for the purpose of the inquiry rather than empirical generalization (Patton, 2002). However, the relevance of our findings for other settings could be considered by countries with similar characteristics.

Although the number of interviewees was limited, the participants had the appropriate competence and practice experience to reflect on the explored topics. It was also possible to reveal a large amount of information and several challenges for good diabetes care in the Omani health-care setting that have not been identified in previous studies.

A possible limitation is that changes in the setting from the time of data collection to date might affect transferability of the findings. However, there were no changes in the guidelines for diabetes care during this period. Another limitation could be related to the background of the principal investigator as a medical doctor employed by the
MoH. Such a connection might either enhance or reduce the credibility (Patton, 2002).

\section{Conclusions and implications}

Our findings implicate a need for an appropriate teamwork approach. The importance of recruiting diabetes specialist nurses in the Omani health-care setting should be emphasized. Clearly defined professional roles and appropriate education to support patients to be able to have a key role in their own care could be useful as the next step to develop diabetes services at primary care level in Oman. Successful implementation could lead to a decrease of the workload, reduction of the frequency of visits to the health centers, improved metabolic control and thereby a decrease in morbidity and mortality due to diabetes complications.

A discussion of our findings among the policy and decision makers in the MoH in Oman could lead to suggestions to strengthen the organization of diabetes care to support patients' needs, and thereby raise the quality of care and health outcomes. The findings further reflect the need for changes in professional behaviour towards less authoritarian and more patient-centred care, and to recognize cultural influences and the patients' beliefs in order to individualize the care according to each person's needs.

\section{Competing interests}

The authors declare that they have no competing interests. Authors' contributions: N.A., M.A.S. and R.W. designed the study. N.A. conducted the interviews, transcribed and analysed them. K.H. checked the data and analysed it together with N.A. N.A. drafted the manuscript, which was critically reviewed by K.H., M.A.S. and R.W. All authors contributed to the write-up of the study, have read and approved the final manuscript.

\section{Acknowledgements}

This study was supported by the Ministry of Health of the Sultanate of Oman and Karolinska Institutet, Sweden. The opinions, results and conclusions reported in this paper are those of the 
authors only. The funders had no influence on the design and conduct of the study; collection, management, analysis and interpretation of the data; or in preparation, review or approval of the manuscript. The authors thank all the participating doctors and nurses.

\section{References}

Abdulhadi, N., AL-Shafaee, M.A., Ostenson, C.G., Vernby, A. and Wahlstrom, R. 2006: Quality of interaction between primary health-care providers and patients with type 2 diabetes in Muscat, Oman: an observational study. BMC Family Practice 7, 72.

Abdulhadi, N., AL-Shafaee, M., Freudenthal, S., Ostenson, C.G. and Wahlstrom, R. 2007: Patient-provider interaction from the perspectives of type 2 diabetes patients in Muscat, Oman: a qualitative study. BMC Health Services Research 7, 162 .

Beck, R.S., Daughtridge, R. and Sloane, P.D. 2002: Physician-patient communication in the primary care office: a systematic review. Journal of the American Board of Family Practice 15, 25-38.

Campbell, J.L., Ramsay, J. and Green, J. 2001: Practice size: impact on consultation length, workload, and patient assessment of care. British Journal of General Practice 51, 644-50.

Campos, C. 2007: Addressing cultural barriers to the successful use of insulin in Hispanics with type 2 diabetes. Southern Medical Journal 100, 812-20.

Cramer, J.A. 2004: A systematic review of adherence with medications for diabetes. Diabetes Care 27, 1218-24.

Dalewitz, J., Khan, N. and Hershey, C.O. 2000: Barriers to control of blood glucose in diabetes mellitus. American Journal of Medical Quality 15, 16-25.

Deakin, T., Mcshane, C.E., Cade, J.E. and Williams, R.D. 2005: Group based training for self-management strategies in people with type 2 diabetes mellitus. Cochrane Database Systematic Reviews CD003417.

Donnelly, M.B. and Anderson, R.M. 1990: The role related attitudes of physicians, nurses, and dieticians in the treatment of diabetes. Medical Care 28, 175-79.

Engelgau, M.M., Narayan, K.M., Saaddine, J.B. and Vinicor, F. 2003: Addressing the burden of diabetes in the 21st century: better care and primary prevention. Journal of the American Society of Nephrology 14 (Suppl 2), S88-91.

Griffin, S.J. 1998: Lost to follow-up: the problem of defaulters from diabetes clinics. Diabetic Medicine 15 (Suppl 3), S14-24.

Helman, C.G. 2007: Culture, health and illness, Third edition. London: Butterworth-Heinemann Ltd.

Hjelm, K., Bard, K., Nyberg, P. and Apelqvist, J. 2003: Religious and cultural distance in beliefs about health and illness in women with diabetes mellitus of different origin living in Sweden. International Journal of Nursing Studies $40,627-43$.
Hjelm, K., Nyberg, P., Isacsson, A. and Apelqvist, J. 1999: Beliefs about health and illness essential for self-care practice: a comparison of migrant Yugoslavian and Swedish diabetic females. Journal of Advanced Nursing 30, 1147-59.

Hjelm, K.G., Bard, K., Nyberg, P. and Apelqvist, J. 2005: Beliefs about health and diabetes in men of different ethnic origin. Journal of Advanced Nursing 50, 47-59.

Insight Guides. 2009: Oman \& The UAE. London: APA Publications.

Karliner, L.S., Jacobs, E.A., Chen, A.H. and Mutha, S. 2007: Do professional interpreters improve clinical care for patients with limited English proficiency? A systematic review of the literature. Health Services Research 42, 727-54.

Lawrence, P. and Rozmus, C. 2001: Culturally sensitive care of the Muslim patient. Journal of Transcultural Nursing 12, 228-33.

Lewis, K., Patel, D., Yorston, D. and Charteris, D. 2007: A qualitative study in the United Kingdom of factors influencing attendance by patients with diabetes at ophthalmic outpatient clinics. Ophthalmic Epidemiology 14, 375-80.

Ministry of Health (MoH). 2003: Diabetes Mellitus Management Guidelines for Primary Health Care, Second edition. Muscat, Oman: Ministry of Health.

MoH. 2008: World Health Survey. Muscat, Oman: Ministry of Health.

Modak, M., Dixit, P., Londhe, J., Ghaskadbi, S. and Devasagayam, T.P. 2007: Indian herbs and herbal drugs used for the treatment of diabetes. Journal of Clinical Biochemistry and Nutrition 40, 163-73.

Parchman, M.L., Pugh, J.A., Noel, P.H. and Larme, A.C. 2002: Continuity of care, self-management behaviors, and glucose control in patients with type 2 diabetes. Medical Care 40, 137-44.

Patton, M.Q. 2002: Qualitative evaluation \& research methods, Third edition. Thousand Oaks, California: Sage Publications.

Peters, J., Hutchinson, A., MacKinnon, M., McIntosh, A., Cooke, J. and Jones, R. 2001: What role do nurses play in Type 2 diabetes care in the community: a Delphi Study. Journal of Advanced Nursing 34, 179-88.

Peyrot, M., Rubin, R.R., Lauritzen, T., Skovlund, S.E., Snoek, F.J., Matthews, D.R., Landgraf, R. and Kleinebreil, L. 2005: Resistance to insulin therapy among patients and providers: results of the cross-national Diabetes Attitudes, Wishes, and Needs (DAWN) Study. Diabetes Care 28, 2673-79.

Puder, J.J. and Keller, U. 2003: Quality of diabetes care: problem of patient or doctor adherence? Swiss Medical Weekly 133, 530-34.

Renders, C.M., Valk, G.D., Griffin, S.J., Wagner, E.H., Eijk Van, J.T. and Assendelft, W.J. 2001: Interventions to improve the management of diabetes in primary care, outpatient, and community settings: a systematic review. Diabetes Care 24, 1821-33.

Rethans, J.J., Norcini, J.J., Barón-Maldonado, M., Blackmore, D., Jolly, B.C., LaDuca, T., Lew, S., Page, G.G. and Southgate, L.H. 2002: The relationship between 
competence and performance: implications for assessing practice performance. Medical Education 36, 901-09.

Rhodes, P. and Nocon, A. 2003: A problem of communication? Diabetes care among Bangladeshi people in Bradford. Health and Social Care in the Community 11, 45-54.

Sackett, D.L. 1997: Evidence-based medicine. Seminars in Perinatology 21, 3-5.

Wens, J., Vermeire, E., Royen, P.V., Sabbe, B. and Denekens, J. 2005: GPs' perspectives of type 2 diabetes patients' adherence to treatment: a qualitative analysis of barriers and solutions. BMC Family Practice 6, 20.

Whiting, D.R., Guariguata, L., Weil, C. and Shaw, J. 2011: IDF Diabetes Atlas: global estimates of the prevalence of diabetes for 2011 and 2030. Diabetes Research and Clinical Practice 94, 311-21.
World Health Organization. 2007: People-Centred-Health Care: a policy framework. Western Pacific Region: WHO.

World Health Organization. 2010: Country Cooperation Strategy for WHO and Oman 2010-2015. Regional Office for the Eastern Mediterranean: WHO.

World Health Organization. 2010 (reprinted in 2011): Global Status Report on non-communicable diseases. Geneva: WHO.

World Health Organization on behalf of the European Observatory on Health Systems and Policies. 2008: Managing chronic conditions: experience in eight countries. WHO Regional Office for Europe: WHO.

World Medical Association. 2009: Medical Ethics Manual, Second edition. Ferney-Voltaire Cedex, France: World Medical Association.

\section{Appendix 1. Questions asked during the interviews}

Thank you for being willing to take part in this interview. I would like first to assure you that no records of this interview would be kept with your name on them.

1 - Could you please start by describing an adult patient with type 2 diabetes you have seen recently?

Probes:

- Please tell me what happened when this patient came to you?

- Could you describe what you discussed when this patient consulted you?

- What did you tell this patient about his/her health condition?

- Did you experience any difficulties in the communication and interaction with this patient?

2 - In general, do you experience difficulties in communication with patients with type 2 diabetes? If difficulties are present, the following two questions are:

$2 a-$ Will you please explain the types of difficulties?

$2 b-$ How do you deal with such difficulties?

3 - Are you usually able to see solutions to problems and difficulties when dealing with patients with type 2 diabetes?

4 - What are your suggestions to achieve optimum interaction with patients with type 2 diabetes in the future?

Primary Health Care Research \& Development 2013; 14: 258-269 


\section{Appendix 2. Examples of the coding during qualitative content analysis}

\begin{tabular}{|c|c|c|c|}
\hline Meaning unit & Code/codes & Sub-category & Category \\
\hline $\begin{array}{l}\text { Yes, mainly some patients being on } \\
\text { maximum dosage of tablets, but the } \\
\text { glycaemic control is not good. I added } \\
\text { glitazone to her old medication but } \\
\text { she did not use it. I want to start } \\
\text { insulin but there is a reluctance to } \\
\text { use the insulin. It seems to be there } \\
\text { are some beliefs among patients } \\
\text { that if they are on insulin that is the } \\
\text { end of it, it's like a terminal case so } \\
\text { they don't accept that. We reinforce } \\
\text { changes in lifestyle, and just keep } \\
\text { on waiting and hoping. }\end{array}$ & $\begin{array}{l}\text { - Maximum dose anti- } \\
\text { diabetes tablets } \\
\text { - Poor glycaemic control } \\
\text { - Reluctance to add new } \\
\text { medicine } \\
\text { - Reluctance to start } \\
\text { insulin } \\
\text { - Beliefs about insulin }\end{array}$ & $\begin{array}{l}\text { Poor patient } \\
\text { adherence to } \\
\text { medicine }\end{array}$ & Patient factors \\
\hline $\begin{array}{l}\text { I and the nurse discussed with } \\
\text { her many times about the use and } \\
\text { benefit of prescribing anti-diabetes } \\
\text { medicines and prevention of } \\
\text { complications. We do our best but } \\
\text { the patients do not listen and do not } \\
\text { understand. I am fed up and also } \\
\text { I threatened her in the last visit that } \\
\text { I will not prescribe any medicine } \\
\text { again since she is not using them; } \\
\text { then the patient was shocked and } \\
\text { promised to be compliant. }\end{array}$ & $\begin{array}{l}\text { - Explanation of benefit of } \\
\text { using anti-diabetes } \\
\text { medicine } \\
\text { - Provision of care and } \\
\text { health education to the } \\
\text { patients } \\
\text { - Doctor is fed up } \\
\text { - Threatening the patients }\end{array}$ & $\begin{array}{l}\text { Doctors' and nurses' } \\
\text { frustration }\end{array}$ & $\begin{array}{l}\text { Factors related to } \\
\text { health-care providers }\end{array}$ \\
\hline
\end{tabular}

